\title{
BMJ Open How do general practitioners access guidelines and utilise electronic medical records to make clinical decisions on antibiotic use? Results from an Australian qualitative study
}

\author{
Ruby Biezen, ${ }^{\oplus 1}$ Cassandra Roberts, ${ }^{1}$ Kirsty Buising, ${ }^{2,3}$ Karin Thursky, ${ }^{\oplus 2}$ \\ Douglas Boyle, ${ }^{1}$ Phyllis Lau, ${ }^{1}$ Malcolm Clark, ${ }^{1}$ Jo-Anne Manski-Nankervis ${ }^{\oplus}$
}

To cite: Biezen R, Roberts $C$, Buising K, et al. How do general practitioners access guidelines and utilise electronic medical records to make clinical decisions on antibiotic use? Results from an Australian qualitative study. BMJ Open 2019;9:e028329. doi:10.1136/ bmjopen-2018-028329

- Prepublication history and additional material for this paper are available online. To view these files, please visit the journal (http://dx.doi.org/10. 1136/bmjopen-2018-028329).

Received 03 December 2018 Revised 17 June 2019 Accepted 16 July 2019

D Check for updates

(C) Author(s) (or their employer(s)) 2019. Re-use permitted under CC BY-NC. No commercial re-use. See rights and permissions. Published by BMJ.

${ }^{1}$ Department of General Practice, The University of Melbourne, Carlton, Victoria, Australia

${ }^{2}$ National Centre for

Antimicrobial Stewardship,

The University of Melbourne,

Melbourne, Victoria, Australia

${ }^{3}$ Victorian Infectious Diseases

Serivce, The Royal Melbourne

Hospital, Melbourne, Victoria,

Australia

Correspondence to

Ruby Biezen;

ruby.biezen@unimelb.edu.au

\section{ABSTRACT}

Objective This study aimed to explore how general practitioners (GPs) access and use both guidelines and electronic medical records (EMRs) to assist in clinical decision-making when prescribing antibiotics in Australia. Design This is an exploratory qualitative study with thematic analysis interpreted using the Theory of Planned Behaviour (TPB) framework.

Setting This study was conducted in general practice in Victoria, Australia.

Participants Twenty-six GPs from five general practices were recruited to participate in five focus groups between February and April 2018.

Results GPs expressed that current EMR systems do not provide clinical decision support to assist with antibiotic prescribing. Access and use of guidelines were variable. GPs who had more clinical experience were less likely to access guidelines than younger and less experienced GPs. Guideline use and guideline-concordant prescribing was facilitated if there was a practice culture encouraging evidence-based practice. However, a lack of access to guidelines and perceived patients' expectation and demand for antibiotics were barriers to guidelineconcordant prescribing. Furthermore, guidelines that were easy to access and navigate, free, embedded within EMRs and fit into the clinical workflow were seen as likely to enhance guideline use.

Conclusions Current barriers to the use of antibiotic guidelines include GPs' experience, patient factors, practice culture, and ease of access and cost of guidelines. To reduce inappropriate antibiotic prescribing and to promote more rational use of antibiotic in the community, guidelines should be made available, accessible and easy to use, with minimal cost to practicing GPs. Integration of evidence-based antibiotic guidelines within the EMR in the form of a clinical decision support tool could optimise guideline use and increase guideline-concordant prescribing.

\section{INTRODUCTION}

Antibiotics are essential for both treatment and prevention of bacterial infections, ${ }^{1}$ but their efficacy is increasingly threatened by

\section{Strengths and limitations of this study}

- The use of qualitative methodology allowed the collection of rich and complex data, offering important insights into how general practitioners access and use guidelines and electronic medical records to assist in clinical decision-making when prescribing antibiotics in Australia.

- In addition, the use of a theoretical framework to underpin the thematic analysis strengthened the transferability of our findings.

- Participants had a general interest in improving guideline-concordant antibiotic prescribing, which might have led to selection bias.

the emergence of bacterial resistance, which can render these drugs ineffective. ${ }^{2} 3$ One of the leading drivers of antibiotic resistance is the amount of antibiotics used. ${ }^{4}$ Without interventions to reduce antibiotic prescribing and usage, it is estimated that, by 2050, 10 million people will die from once treatable infections. ${ }^{5}$ Australia was ranked sixth highest in terms of rates of antibiotic prescribing per capita in the community compared with 28 European countries. ${ }^{6}$ Prescriptions issued in the community are a significant contributor, with general practitioners (GPs) generating nearly 9 in 10 prescriptions for antibiotics. ${ }^{6}$

Antibiotics continue to be prescribed inappropriately. ${ }^{78}$ A high proportion of antibiotic prescriptions in the Australian community are being issued for conditions that should not typically require antibiotic therapy, such as upper respiratory tract infections, ${ }^{6}$ which are usually caused by viruses. Recent studies have described barriers to promoting more rational use of antibiotics, including patients' misconceptions regarding the effectiveness of antibiotics for certain indications, physicians' 
perceptions of patient pressure to prescribe antibiotics, diagnostic uncertainty and patients' expectation for quick fixes for illness. ${ }^{9-13}$ These factors may divert practice from recommended guidelines. Access to antibiotic guidelines may be another reason. For example, the Royal Australian College of General Practitioners' (RACGP) Standards indicate that the use of Therapeutic Guidelines (TG), the recommended guideline in Australia, is considered standard practice. ${ }^{14}$ However, it is only available to GPs via a paid subscription which may be a barrier limiting uptake in some settings. It is not clear whether GPs are accessing TG or other guidelines to inform their decision-making and prescribing of antibiotics. To promote the rational use of antibiotics, it is imperative that evidence-based guidelines are available, accessible and adhered to as they provide clear, practical, succinct and up-to-date information to assist decision-making around antibiotic prescribing. ${ }^{14}$

In the Australian general practice setting, antibiotics are usually prescribed within electronic medical records (EMRs). EMRs facilitate timely access to relevant clinical patient information that is easy to read, the recording of patient clinical information in real-time and some provide prompts and alerts. ${ }^{15}$ Embedding access to information from guidelines within EMRs may be a strategy to assist GPs' decision-making when prescribing antibiotics. Currently it is not clear whether GPs are accessing guidelines, such as TG, and how they are using these to inform their decision-making and prescribing of antibiotics. Hence, the aim of the study was to explore how GPs access and use guidelines in Australia, and whether incorporating guidelines within EMR could assist in clinical decision-making when prescribing antibiotics.

\section{METHODS}

\section{Study design}

An exploratory qualitative study design was used to understand GPs' access and use of guidelines and EMRs in general practice to inform antibiotic prescribing. Focus groups using a semistructured interview schedule (online supplementary appendix 1) were conducted with GPs in
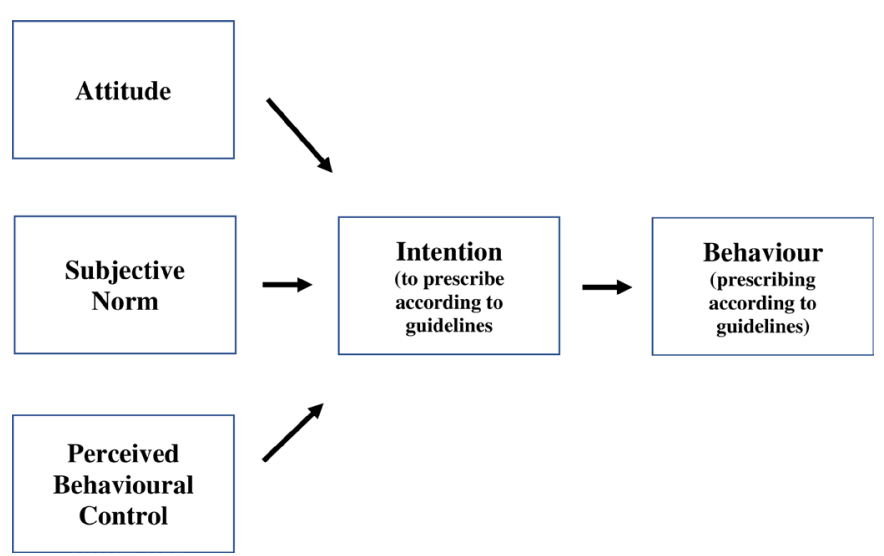

Figure 1 Ajzen's Theory of Planned Behaviour. ${ }^{16}$ five general practices. For validity, focus group questions were piloted with two GPs. Data from the pilot interviews were not included in the final analysis.

\section{Study recruitment}

Five general practices were recruited in Victoria, Australia, to participate in this study. The practices were recruited through the investigators' professional networks. The only inclusion criteria were that practices had to be using computerised EMRs to record patient consultations. The practices were contacted and a practice visit was organised by $\mathrm{RB}$ where the research plan was discussed with the practice manager and the principal GP. Participants were informed that the focus group (up to an hour duration) would be conducted by a final year medical student (CR) and facilitated by an experienced researcher (RB). All participants completed the consent form before the commencement of the focus group. Follow-up calls were made to organise the focus group with the practice if a time for the focus group had not been finalised at the time of recruitment. Participants were reimbursed for their time with a free 12-month subscription to TG (valued at \$A367) on completion of the focus groups.

\section{Data collection}

Four focus groups were conducted in-person at the participating general practices and one was conducted via video conferencing between February and April 2018. One additional practice expressed an interest to participant in the study; however, they did not have the minimum required GPs to conduct a focus group. Data from the focus groups were digitally recorded and transcribed verbatim. At the end of each focus group, the researchers met to discuss the outcomes and discussed the important areas and emerging themes. These emerging themes were fine-tuned and became part of the 'prompts' for subsequent focus groups. Transcripts were reviewed to remove any identifying information, and all GP and practice names were replaced by a pseudonym or code. All transcripts were read at least twice and compared with the recording for accuracy.

\section{Data analysis}

Ajzen's Theory of Planned Behaviour (TPB) was used to provide a framework to analyse the results. This theory states that attitudes, subjective norms and perceived behavioural control together influence a person's intentions and their subsequent behaviour (figure 1), that is, a person's behaviour is influenced by the attitude, perceived social pressure (subjective norm) and the extent to which a person feels they are in control of performing a certain behaviour (perceived behaviour control). ${ }^{16}$ This structured framework has been used in previous studies to understand barriers and facilitators to screening, ${ }^{17} 18$ preventive care behaviour, ${ }^{19}$ medication prescribing $^{20}$ and behaviour to treatment pathways ${ }^{21}$ in the general practice setting. 
Two researchers (RB and CR) independently coded the first transcript to identify pertinent concepts and ideas. A coding scheme was derived by grouping emerging concepts during data collection and early analysis. Using a thematic analysis approach ${ }^{22}$ codes were then merged and grouped to form themes and subthemes. The coding schemes from both researchers were then compared and refined. Differences were resolved by negotiation and consensus, and consultation with a third researcher (JAMN). This process was repeated until all transcripts were coded. The codes and themes were then matched to the domains within the TPB framework. Data were managed using NVivo V.11 (QSR International, Doncaster, Australia).

\section{Patient and public involvement}

Our study was an exploratory study on how GPs access and use guidelines and EMRs to make clinical decisions on antibiotic use; hence, no patient participants were involved at this stage. However, patient participants will be involved in future phases of the study, as clinical decision-making for antibiotic prescribing directly affects, and is influenced by, patients.

\section{RESULTS}

A total of 26 GPs from five general practices participated in the five focus groups. Focus groups consisted of three to eight GPs, with an average duration of $36 \mathrm{~min} 25 \mathrm{~s}$. Fourteen (54\%) GPs were male. The GPs had an average age of 40 (range 28-68) years and an average of 11 (range from 1 to $>30$ ) years of clinical experience. One practice was classified being located in inner regional Australia, while the other four practices were classified as major city according to the Australian Bureau of Statistics. ${ }^{23}$

Themes were grouped within the TPB framework into attitudes, subjective norms and perceived behavioural control (figure 2). An additional theme about the use of the EMR to provide clinical decision support was included as this did not clearly map to a TBP component. We elaborate on these intentions and behaviour below. Each theme was supported by quotes indexed by focus

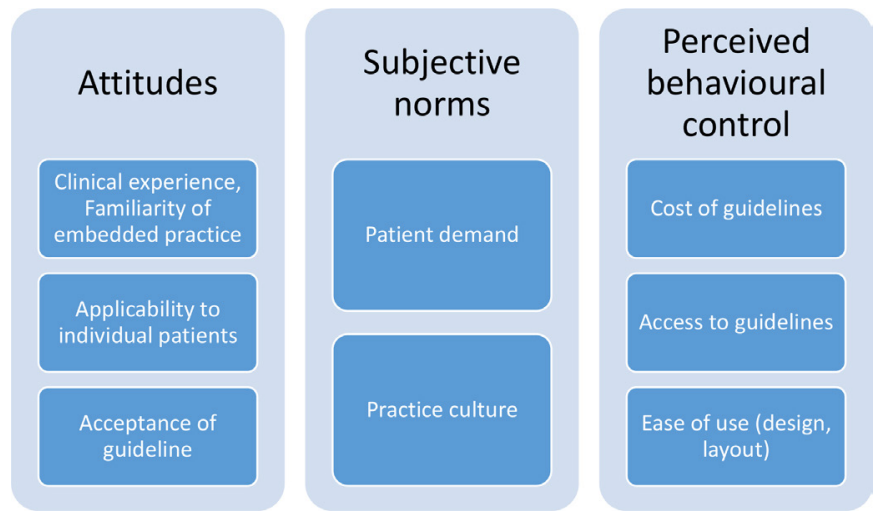

Figure 2 Key themes mapped to Ajzen's Theory of Planned Behaviour. group number, as well as the participants' gender and years of clinical experience, for example, focus group (FG) 1, M (male), >15 years. Two researchers (RB and CR) determined independently that no new themes or perspectives were emerging after analysis of the fourth focus group indicating that data saturation had occurred; this was confirmed on analysis of the fifth focus group.

\section{Attitude - use of guidelines}

GPs agreed guidelines play an important role in providing standardised care to patients. It was seen as being able to address any clinical questions GPs might have during a consultation:

'I think guidelines are good because we are after consistency in prescribing antibiotics and consistency means that we're all doing our part in terms of stewardship and hopefully decreasing the chance of microbial resistance... it's quickly accessible, and I can really quickly access the clinical question I have. Because sometimes it's just a clinical question. I'm thinking I want to know what the right dose is. I may already know the right medication, for example, a 10-year-old child with a sore throat has got a high risk of rheumatic fever... penicillin if there isn't any allergy, right. So I know the antibiotic... but I may just need to know the dose, so something that I can quickly access the actual clinical question that I have.'FG2, M, 5-15 years

'My experience has been that they're good. I suppose there is those times where you don't necessarily give antibiotics all that often I'd probably go and check. My experience has been that I usually get the information I need from them.'-FG5, F, 5-15 years

However, willingness to refer to guidelines appeared to be influenced by GPs' clinical experience: GPs who had fewer years of experience reported using TG regularly, while more experienced GPs expressed that they tended to 'know' what to prescribe already. Recognition of individual patient needs including their perceived preference for antibiotics influenced GPs' prescribing decisions, which could lead to diversion from guideline recommendations.

'The more experience you get the less you probably access [guidelines].'-FG1, F, $<5$ years

'The guidelines are not black and white...they're recommendations that sometimes we need to go outside of.'-FG1, M, $>15$ years

'There's an element of knowing the patient or understanding the patient's history that you can't ever put into a guideline. You get some grasp on previous illnesses and previous experiences and a better understanding of the reliability of the patient's history for example, things like that that are a little bit hard to put into a guideline. When Joe Blogs comes in and tells me he's feeling particularly lousy then he's probably dead, whereas when Jill Blogs comes in you don't 
listen to anything she says because she's always dead. So that's where the clinical judgment comes into it a lot as well.'-FG5, M, 5-15 years

With experience, doctors perceived that they already knew the guideline recommendations for common conditions (such as urinary tract infections) which limited guideline use. Furthermore, some GPs have their 'preferred antibiotics', and as long as the condition was not complex, GPs would use their 'default' prescriptions rather than checking a guideline.

'It makes me realise how few times I'm looking [the guidelines] up actually...You just go to your favourites (antibiotics).'-FG3, F, $>15$ years

Issues around trust and acceptance of guideline recommendations were also mentioned. GPs might prescribe against recommended guidelines if they felt the information was 'outdated'. In addition, guidelines from different bodies that contradicted each other caused confusion as to the best or most appropriate patient management. GPs commented that there was a need for one overarching Australian guideline to increase perceived validity.

'Sometimes the guidelines contradict each other, and so that's when I definitely-well, you're going to go against one of them. Like the absolute cardiovascular risk guidelines, and then your metabolic syndrome guidelines, they don't really overlap. So metabolic syndrome could be diagnosed and the International Diabetes Federation will say 'treat the individual factors which are contributing to that', but the absolute risk might not be high, or even moderate. But we know that metabolic syndrome does make people a high risk, but it's not mentioned in the absolute risk guidelines. So there's things like siloed groups writing things and missing small components that are pretty relevant to that. Maybe they're trying to simplify, but it just makes it confusing for us.' - FG1, F, $<5$ years

\section{Subjective norm-social pressure of guideline use}

Perceived patient demand was reported as a major reason for diverting from guideline recommendations. GPs described situations where patients were insistent that previously prescribed antibiotics caused side effects or 'did not work'.

'There's a lot around patient expectations... sometimes you're forced to go against [guidelines].'FG3, $\mathrm{M},<5$ years

'Sometimes, GPs almost get bullied by their patient...'-FG3, M, $<5$ years

'This is the guideline, this patient have this thing, you give this medication. But sometimes the patient will tell you, "No, doctor." Suppose the antibiotic you prescribed in this one, "No, it didn't work for me last time," or, "My friend used it, it didn't work." Sometimes we got this stuff. It's less but we got this confrontation. So we need to explain more... And sometimes however probably some of the doctor have less time, prescribe the antibiotic, we tell it mostly likely due to the virus, antibiotic cannot kill the viruses. If you are giving the antibiotic randomly it will make resistance and... everything.'-FG2, M, >15 years

Culture within practices was reported to strongly influence antibiotic prescribing: practices who reported that GPs shared guideline updates and regularly discussed patient cases said that this reinforced a positive culture of guideline-concordant prescribing. It encouraged communication and support among GPs, which the GPs felt reduced inappropriate prescribing. In contrast, at other practices, GPs reported that they were likely to succumb to patient pressure and prescribe antibiotics. They said they feared patients would seek antibiotics elsewhere and that raising concerns about inappropriate antibiotic prescribing by other doctors in the practice might be seen to be undermining them.

'The problem is that working in a group practice you don't want to be undermining your peers. ' $-F G 3, M,<5$ years

\section{Perceived behavioural control—practical issues affecting guideline use}

The cost of a subscription to access guidelines was also an important consideration reported to affect use. While some practices have a TG subscription that allowed GP access, other GPs reported that they had to subscribe individually. Some GPs said they felt the cost was not only expensive but also inequitable, as doctors from hospitals were provided with access to TG for free. Some GPs expressed the view that the need for a paid subscription meant that the use of guidelines was seen as being 'optional'.

The biggest issue with eTG is cost. So for us as a practice we didn't have it for quite some time because it was going to cost us...'-FG3, $F$, >15 years

'When I'm in the hospital it's free, and when I'm in GP training it's free [but] once I became a fellow it's very expensive to get it by paying by myself so I didn't use Therapeutic Guidelines...'-FG4, F, $<5$ years

'What would be amazing, it is in your fellowship fees every year, you got a subscription that said to you, "We want you to stay up to date, we are going to encourage you", therefore TG is free.'-FG3, M, $<5$ years

Concerns about the design and layout were also reported to hinder GPs' use. For examples, search engines requiring exact phrasing, convoluted navigation and too much information were reported problems.

'It has everything in it and you have to find the bit that's relevant to you. It will tell you what to do if someone's an ATSI patient coming down from Darwin, it's going to tell you what to do if someone's in a tropical environment, it's going to tell you what 
to do in a hospital, and then also, and it's just trying to find which one of those is relevant to you. And sometimes you read it and you're just skimming and you're like, "Oh yeah, I'll give azithromycin," and then you look back a bit later and you go, "Oh, that was if they were blah, blah. Oops, I should have done something else". So sometimes there's just too much information.'-FG3, M, $<5$ years

'I'm just thinking of Therapeutic Guidelines and sometimes the index isn't as simple as it could be.'FG5, F, 5-15 years

'Recently I had a case of I think diverticulitis that I decided I'd look up again. Most conditions we're prescribing antibiotics for are the same routinely; urinary tract infections and skin infections and respiratory tract infections. I would never consult a guideline primarily because I assume I know them by this stage, unless it's something really unusual going on. But I did recently pull up the therapeutic guidelines just to read through the latest on diverticulitis, which confused me more and I think I did what I was going to do in the first place.'-FG5, M, 5-15 years

Ease of access was another factor which influenced the use of guidelines. For instance, accessing a separate browser, a requirement to login each time, and keeping track of passwords were seen as unnecessary burdens that added time to an already time constraint consult.

'TG can be cumbersome: you've got to open a web browser, put your password in, and then search.'FG1, $\mathrm{F},<5$ years

Some GPs were concerned there was a perception that accessing guidelines through an internet browser might result in patients perceiving that the GP was not competent.

'Using a web browser doesn't look like you know what you're doing.' - FG1, $\mathrm{F},<5$ years

In general, features that were seen as 'user-friendly' and would increase guideline use included flow diagrams with summarised information, and platforms that were easy to navigate.

\section{Accessing guidelines within EMR for decision support?}

The EMRs used by the GPs did not provide clinical decision support to assist with antibiotic prescribing; GPs commented that embedding guidelines within the EMR would be a helpful initiative that could lead to guideline concordance.

'The software doesn't really help you decide what to prescribe, but it will have a history of what they've had before. Sometimes they'll say "oh, that last one worked well" or whatever. Sometimes it's not overly useful, perhaps not guideline recommended...'FG1, $\mathrm{F},<5$ years
'If TG could be in Best Practice [EMR] that would be amazing.' $-\mathrm{FG} 1, \mathrm{~F},<5$ years

Participants also commented that the current workflow in the EMR did not require documentation of prescribing rationale. While the option exists, it was not compulsory to enter a reason for prescription. Including the rationale for prescribing will further justify the need to prescribe and encourage guideline compliance.

'There is an option to choose reason for prescription, but I certainly never use it and I've never seen anybody use it within the clinic.'-FG5, M, 5-15 years

\section{DISCUSSION}

This study highlighted that decision-making around antibiotic prescribing and whether guidelines were accessed depend on multiple factors such as GPs' clinical experience; their knowledge and perception of individual patient expectations; trust and acceptance of guidelines; social influences from peers; as well as guideline software design, accessibility and cost. Currently, EMRs do not provide clinical decision support to assist with antibiotic prescribing, and guidelines can only be accessed via the internet external to the EMR system.

Attitudes towards guidelines was an important factor influencing our participants' guideline use. GPs with less clinical experience and knowledge of the guidelines would access guidelines more often, but guidelines that did not account for individual patients' circumstances were less likely to be adhered to. Interestingly more experienced GPs did not perceive any need to check guidelines for updated evidence, but rather seemed comfortable with familiar patterns of prescribing. Our findings are consistent with study outcomes from the UK, Europe and New Zealand. ${ }^{24-28}$ For instance, a systematic review and metasynthesis of 12 qualitative studies from countries including UK, Europe, USA and Canada found that GPs' clinical experience and knowledge of the guideline impacted on whether GPs adhered to clinical guidelines. ${ }^{25}$ Similarly, a systematic review by Farquhar et al found 30\% of clinicians thought guidelines were impractical and did not always accommodate individual patients, and $34 \%$ thought guidelines reduced their autonomy and oversimplified medicine. ${ }^{28}$ Importantly not all guidelines are the same, and the use of a given guideline can be highly variable. While GPs from our studies agreed that guidelines were useful, it did not seem to translate to everyday use. Addressing these concerns and involving GPs in the development of guidelines may increase acceptance of robust and evidence-based guidelines, and encourage its use.

Perceived patient demand and expectations was reported to be a major barrier to guideline use. Previous research has demonstrated that GPs believe patients often have an expectation of antibiotics before coming for a consultation, ${ }^{29-31}$ or previous negative experiences (such as side effects) from particular antibiotics which the GPs said 
forced them to prescribe outside recommended guidelines. ${ }^{25-28}$ While guideline recommendations should be considered, GPs' experience of patient demand should not be downplayed. Therefore, educating patients through public awareness campaigns on the effectiveness of antibiotics may reduce expectations for certain illnesses ${ }^{32}$ which, in turn, may reduce GPs' perception of patient demands, ${ }^{33}$ leading to an increase in guideline compliance and better patient health outcomes.

Practice culture was an important factor highlighted by GPs in this study. An organisation that values evidencebased practice, makes guidelines available and discusses prescribing made it easier for GPs to prescribe in a guideline compliant way. The value of creating and supporting this culture, and its impact on individual GP practice was a notable finding.

Barriers such as guideline design and layout, ease of access and whether it fitted into the clinical workflow were common barriers to guideline use noted in this study, which is consistent with published literature. ${ }^{25} 27$ Guidelines that are difficult to navigate, accessed through multiple web pages and having more than three 'clicks' are usually considered to be unwieldly and add to an otherwise time constrained consultation. Hence guidelines need to be intuitive, evidence-based and easy to access in order to increase the likelihood of them being used.

Our study suggested cost was an important factor influencing GPs' guideline use. In 2018, the Antimicrobial Stewardship in Australian Healthcare strategy endorsed the use of TG and stating that its use is an essential element of stewardship programme. ${ }^{34}$ In fact, the main guideline use in Australia for antibiotic prescribing is Therapeutic Guidelines: Antibiotic. ${ }^{35}$ These guidelines provide clear, practical, succinct and up-to-date information to assist clinicians in decision-making around antibiotic prescribing. However, TG is only accessible via a paid subscription for GPs. In order to encourage GPs to access and use TG, strategies to address cost are likely to be required. The increased accessibility of guidelines and streamlining GP workflow may facilitate practice policy and drive development of a culture to prescribing in line with evidence-based guidelines.

Studies have demonstrated that clinical decision support tools, together with evidence-based guidelines, improve adherence to guidelines and better disease management. ${ }^{36-38}$ For example, a cluster randomised trial with 43 primary care clinics found a significant increase in guideline adherence regarding anticoagulant therapy in the clinical decision support group $(\mathrm{p}=0.013)$ compared with the control group, with a treatment effect estimate of 0.016 [95\% CI 0.003 to 0.028 ].$^{36}$ Similarly, a study of 235 patients with Down syndrome found selected recommendations for Down syndrome care integrated into the electronic health records was associated with a shift in adherence from $61.6 \%$ to $77.3 \%$ ( $\mathrm{p}<0.001) .{ }^{37}$ Therefore it is important to consider integrating evidence-based guidelines, in the form of a clinical support tool, to assist access to guidelines and improve workflow, leading to better patient outcome.
One of the strengths in our study was the use of a theoretical framework, the Theory of Planned Behaviour, to underpin our thematic analysis. This provided a valid and evidence-based approach where barriers identified can inform interventions to assist GPs' decision-making process when prescribing antibiotics. Although our participants were enthusiastic to share their experience and ideas which enriched our data, this might have also led to possible study bias which is a limitation. In addition, our study did not target specific conditions, hence the variation of guideline use for different conditions was not explored. Supplying a free 12-month subscription to TG as an incentive might have also influenced how GPs responded to our questions resulting in selection bias. Nonetheless, this incentive potentially encouraged GP use of TG which is in line with recommendations from RACGP quality standards. ${ }^{14}$

The results of our study suggest that a multifactorial approach should be considered to overcome some of the barriers to the use and access guidelines to inform antibiotic prescribing. Previous studies have indicated that incorporating clinical decision support tools including guidelines into electronic health records is likely to assist guideline adherence and provide better health outcomes. ${ }^{36}{ }^{37}$ As over $90 \%$ of GPs use a clinical software package in their practice ${ }^{15}$ incorporating guidelines into electronic record will be a positive step forward. For this to be successful, we need to involve GPs and other end users in the development of guidelines to ensure usability and acceptability of the product, and embed this into the clinical workflow. ${ }^{39} 40$ In addition, cost to access guidelines need to be addressed, either through financial incentives or low cost or even free access to TG for all Australian GPs to encourage GPs to comply to quality standards when prescribing. Finally, public health and information campaigns to increase antibiotic resistance knowledge and educate the community on the judicious use of antibiotics are important to reduce patient demand for inappropriate antibiotic prescribing. ${ }^{41-43}$

\section{CONCLUSIONS}

Key barriers to the access and use of antibiotic guidelines in Australian general practice include familiarity of embedded practice, needs of individual patients and perceived pressure from patients to prescribe against guidelines. Cost, design and format, and the need to access guidelines separately from the EMR were also barriers to their use in general practice. It was important to GPs that an endorsed set of prescribing guidelines existed. They would be more likely to use them if access fitted workflow. The integration of antibiotic guidelines within the EMR in the form of a clinical decision support tool could address several key barriers to their use, assist with decision-making and improve the prescribing of antibiotics. 
Acknowledgements We would like to thank all the GPs who participated in this research. The researchers gratefully acknowledge Therapeutic Guidelines and The Royal Australian College of General Practitioners Foundation for their support of this project.

Contributors JAMN completed the background literature search and JAMN, $\mathrm{KB}, \mathrm{KT}, \mathrm{DB}, \mathrm{PL}$ and $\mathrm{MC}$ contributed to the study design. $\mathrm{RB}$ and $\mathrm{CR}$ conducted all interviews. RB, CR and JAMN performed the analysis of the data. RB and CR drafted the manuscript. All authors revised all drafts and approved the final version of the manuscript.

Funding This work was supported by The Royal Australian College of General Practitioners (RACGP) grant number TGL 17565484.

Competing interests None declared.

Patient consent for publication Not required.

Ethics approval Minimal risk ethics project approval was granted by the Department of General Practice Human Ethics Advisory Group, The University of Melbourne (Ethics ID: 1750248.1).

Provenance and peer review Not commissioned; externally peer reviewed. Data availability statement Data are available on reasonable request.

Open access This is an open access article distributed in accordance with the Creative Commons Attribution Non Commercial (CC BY-NC 4.0) license, which permits others to distribute, remix, adapt, build upon this work non-commercially, and license their derivative works on different terms, provided the original work is properly cited, appropriate credit is given, any changes made indicated, and the use is non-commercial. See: http://creativecommons.org/licenses/by-nc/4.0/.

\section{REFERENCES}

1. Australian Government. Responding to the threat of antimicrobial resistance: Australia's First National Antimicrobial Resistance Strategy 2015-2019. Australian Government. Available: https://www. amr.gov.au/resources/national-amr-strategy [Accessed 16 Nov 2018]

2. World Health Organization. The evolving threat of antimicrobial resistance. options for action. World Health Organization, 2012.

3. Centers for Disease Control and Prevention. Antibiotic resistance threats in the United States, 2013. U.S. Department of Health and Human Services, 2013.

4. Austin DJ, Kristinsson KG, Anderson RM. The relationship between the volume of antimicrobial consumption in human communities and the frequency of resistance. Proc Natl Acad Sci U S A 1999:96:1152-6.

5. O'Neill J. Antimicrobial Resistance: Tackling a crisis for the health and wealth of nations. The Review on Antimicrobial Resistance, 2014.

6. Australian Commission on Safety and Quality in Health Care. AURA 2016: First Australian report on antimicrobial use and resistance in human health. Sydney: ACSQHC, 2016.

7. McCullough AR, Pollack AJ, Plejdrup Hansen M, et al. Antibiotics for acute respiratory infections in general practice: comparison of prescribing rates with guideline recommendations. Med J Aust 2017;207:65-9.

8. Gerding DN. The search for good antimicrobial stewardship. Jt Comm J Qual Improv 2001;27:403-4.

9. Belongia EA, Naimi TS, Gale CM, et al. Antibiotic use and upper respiratory infections: a survey of knowledge, attitudes, and experience in Wisconsin and Minnesota. Prev Med 2002;34:346-52.

10. Moro ML, Marchi M, Gagliotti C, et al. Why do paediatricians prescribe antibiotics? results of an Italian regional project. $B M C$ Pediatr 2009;9:69.

11. Mangione-Smith R, Elliott MN, Stivers T, et al. Ruling out the need for antibiotics: are we sending the right message? Arch Pediatr Adolesc Med 2006;160:945-52.

12. Mangione-Smith R, McGlynn EA, Elliott MN, et al. Parent expectations for antibiotics, physician-parent communication, and satisfaction. Arch Pediatr Adolesc Med 2001;155:800-6.

13. Biezen R, Brijnath $B$, Grando $D$, et al. Management of respiratory tract infections in young children-A qualitative study of primary care providers' perspectives. npj Prim Care Resp Med 2017;27.

14. Royal Australian College of General Practitioners. Standards for general practices (5th edition), 2018. Available: https://www.racgp. org.au/your-practice/standards/standards-for-general-practices [Accessed 4 Oct 2018].

15. Mclnnes DK, Saltman DC, Kidd MR. General practitioners' use of computers for prescribing and electronic health records: results from a national survey. Med J Aust 2006;185:88-91.
16. Ajzen I. The theory of planned behavior. Organ Behav Hum Decis Process 1991;50:179-211.

17. Sinclair PM, Day J, Levett-Jones T, et al. Barriers and facilitators to opportunistic chronic kidney disease screening by general practice nurses. Nephrology 2017;22:776-82.

18. Wade J, Smith $\mathrm{H}$, Hankins $\mathrm{M}$, et al. Conducting oral examinations for cancer in general practice: what are the barriers? Fam Pract 2010;27:77-84

19. Ampt AJ, Amoroso C, Harris MF, et al. Attitudes, norms and controls influencing lifestyle risk factor management in general practice. $B M C$ Fam Pract 2009;10:59.

20. Lionis C, Petelos E, Shea S, et al. Irrational prescribing of over-thecounter (OTC) medicines in general practice: testing the feasibility of an educational intervention among physicians in five European countries. BMC Fam Pract 2014;15:34.

21. Kramer L, Schlößler K, Träger S, et al. Qualitative evaluation of a local coronary heart disease treatment pathway: practical implications and theoretical framework. BMC Fam Pract 2012;13:36.

22. Braun V, Clarke V. Using thematic analysis in psychology. Qual Res Psychol 2006;3:77-101.

23. Australian Bureau of Statistics. The Australian statistical geography standard remoteness structure, 2018. Available: http://www.abs. gov.au/websitedbs/D3310114.nsf/home/remoteness+structure [Accessed 15 Nov 2018].

24. Teixeira Rodrigues A, Roque F, Falcão A, et al. Understanding physician antibiotic prescribing behaviour: a systematic review of qualitative studies. Int J Antimicrob Agents 2013;41:203-12.

25. Carlsen B, Glenton C, Pope C. Thou shalt versus thou shalt not: a meta-synthesis of GPs' attitudes to clinical practice guidelines. $\mathrm{Br} \mathrm{J}$ Gen Pract 2007:57:971-8.

26. Carlsen B, Norheim OF. "What lies beneath it all?"--an interview study of GPs' attitudes to the use of guidelines. BMC Health Serv Res 2008;8:218.

27. Langley C, Faulkner A, Watkins C, et al. Use of guidelines in primary care--practitioners' perspectives. Fam Pract 1998;15:105-11.

28. Farquhar CM, Kofa EW, Slutsky JR. Clinicians' attitudes to clinical practice guidelines: a systematic review. Med J Aust 2002;177:502-6.

29. Lucas PJ, Cabral C, Hay AD, et al. A systematic review of parent and clinician views and perceptions that influence prescribing decisions in relation to acute childhood infections in primary care. Scand $J$ Prim Health Care 2015;33:11-20.

30. Stivers T, Mangione-Smith R, Elliott MN, et al. Why do physicians think parents expect antibiotics? what parents report vs what physicians believe. J Fam Pract 2003;52:140-8.

31. Fletcher-Lartey S, Yee M, Gaarslev C, et al. Why do general practitioners prescribe antibiotics for upper respiratory tract infections to meet patient expectations: a mixed methods study. BMJ Open 2016:6:e012244.

32. Ancillotti M, Eriksson S, Veldwijk J, et al. Public awareness and individual responsibility needed for judicious use of antibiotics: a qualitative study of public beliefs and perceptions. BMC Public Health 2018;18:1153.

33. Michael CA, Dominey-Howes D, Labbate M. The antimicrobial resistance crisis: causes, consequences, and management. Front Public Health 2014;2.

34. Australian Commission on Safety and Quality in Health Care. Antimicrobial stewardship in Australian health care 2018, 2018. Available: https://www.safetyandquality.gov.au/our-work/healthcareassociated-infection/antimicrobial-stewardship/book/ [Accessed 2 Nov 2018].

35. Therapeutic Guidelines Limited. Therapeutic guidelines: antibiotic, 2018. Available: https://www.tg.org.au/ [Accessed 3 Nov 2018].

36. Karlsson LO, Nilsson S, Bång M, et al. A clinical decision support tool for improving adherence to guidelines on anticoagulant therapy in patients with atrial fibrillation at risk of stroke: a cluster-randomized trial in a Swedish primary care setting (the CDS-AF study). PLoS Med 2018;15:e1002528.

37. Santoro SL, Bartman T, Cua CL, et al. Use of electronic health record integration for Down syndrome guidelines. Pediatrics 2018;142:e20174119.

38. Chiang J, Furler J, Boyle D, et al. Electronic clinical decision support tool for the evaluation of cardiovascular risk in general practice: a pilot study. Aust Fam Physician 2017;46:764-8.

39. Hvitfeldt Forsberg H, Granström E, et al. Co-Designing an eHealth service for the Co-Care of Parkinson disease: explorative study of values and challenges. J MIR Res Protoc 2018;7:e11278.

40. Sabater-Hernández D, Tudball J, Ferguson C, et al. A stakeholder codesign approach for developing a community pharmacy service to enhance screening and management of atrial fibrillation. BMC Health Serv Res 2018;18:145. 
41. Rush L, Patterson C, McDaid L, et al. Communicating antimicrobial resistance and stewardship in the National press: lessons from sepsis awareness campaigns. J Infect 2019;78:88-94.

42. Di Mario S, Gagliotti C, Buttazzi R, et al. Observational prepost study showed that a quality improvement project reduced paediatric antibiotic prescribing rates in primary care. Acta Paediatr 2018;107:1805-9.

43. Hill EM. Decision making about antibiotic use: examining the role of antibiotic resistance knowledge, concern, and previous inappropriate antibiotic use. J Health Commun 2017;10:226-33. 